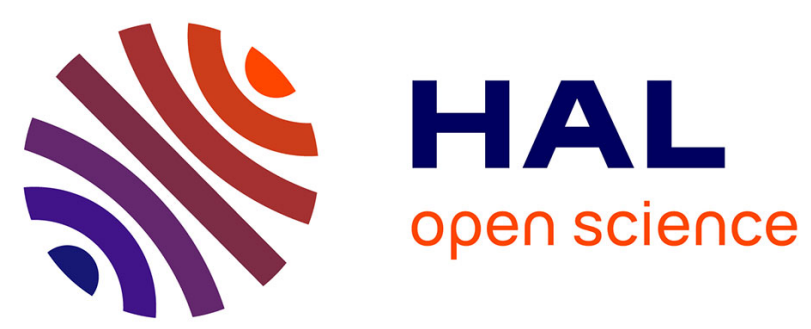

\title{
Décrire les images médiévales
}

\author{
Stavros Lazaris
}

\section{To cite this version:}

Stavros Lazaris. Décrire les images médiévales. Cahiers de la Méditerranée, 1996, 53, pp.149-164. halshs-00005335

\section{HAL Id: halshs-00005335 \\ https://shs.hal.science/halshs-00005335}

Submitted on 8 Nov 2005

HAL is a multi-disciplinary open access archive for the deposit and dissemination of scientific research documents, whether they are published or not. The documents may come from teaching and research institutions in France or abroad, or from public or private research centers.
L'archive ouverte pluridisciplinaire HAL, est destinée au dépôt et à la diffusion de documents scientifiques de niveau recherche, publiés ou non, émanant des établissements d'enseignement et de recherche français ou étrangers, des laboratoires publics ou privés. 
Article paru dans « les Cahiers de la Mediterranée » Actes du IIe colloque national de l'Association Française pour l'Histoire et l'Informatique Décembre 1996, N53 


\title{
DÉCRIRE LES IMAGES MÉDIÉV ALES
}

\author{
Stavros LAZARIS \\ Université de Strasbourg \\ E.H.E.S.S. (Paris)
}

\section{1 - LA SITUATION ACTUELLE DE LA RECHERCHE}

Depuis la fin du siècle dernier, et le regain d'intérêt porté aux oeuvres d'art de l'époque médiévale. les méthodes utilisées n'ont guère évolué. Cette stagnation des méthodes de la recherche a plusieurs causes dont la plus importante était, et continue d'être, la focalisation des médiévistes sur la réunion d'oeuvres d'art de l'époque et surtout sur la publication primaire de celles-ci.

Aujourd'hui les différentes étapes de la recherche dans le domaine de la peinture médiévale (icônes, enluminures, fresques, ...) peuvent être regroupées dans deux catégories d'études : "l'analyse iconographique" et "l'analyse stylistique". Dans celles-ci peuvent d'ailleurs être classés différents travaux de synthèse qui traitent des oeuvres artistiques. Le plus souvent, dans ce genre d'études, la description de l'oeuvre, qui se veut littéraire et globale, figure dispersée dans le texte.

Certes, je n'ai pas l'intention de porter un jugement de valeur sur les travaux relatifs à la peinture médiévale (en effet, ces études sont d'un grand intérêt du fait qu'elles sont les seules capables de fournir, actuellement, une conception historique et globale des oeuvres d'art de cette période). J'aimerais simplement souligner, d'une part des incohérences strictement méthodologiques qui pourraient dans l'avenir être corrigées par une transformation du travail de l'historien de l'art dans l'obligation de répondre à des besoins plus stricts de recherche, et d'autre part un manque cruel d'instruments de travail qui pourraient faciliter la recherche sur les images médiévales.

\section{2 - L'OBJET DE L'ÉTUDE ET SES OBJECTIFS}

Ce travail, dérivé d'une étude sur les rapports texte-image dans les manuscrits grecs scientifiques illustrés, concerne l'image médiévale et plus

(1) - Toutefois, il faut saluer le développement d'importantes entreprises de description, de catalogage ou d'indexation d'images médiévales et rappeler l'existence d'iconothèques pouvant être très utiles aux chercheurs. Malheureusement je suis dans l'obligation de constater que la plupart de ces travaux concernent le monde occidental et qu'en ce qui concerne le monde byzantin, très peu de recherches de ce genre ont vu le jour. 
précisément une de ses formes : la miniature, illustration d'un texte qui a pour but d'expliciter ce demier.

Cette présente étude n’a pas pour ambition de proposer une approche théorique de la miniature médiévale mais au contraire d'établir des principes pour l'élaboration d'un système capable de décrire les images se trouvant dans les manuscrits médiévaux et notamment dans ceux dont le contenu est scientifique.

Car la description est une étape nécessaire qui doit précéder toute construction théorique (typologique ou interprétative) ${ }^{2}$ et qui, en même temps, doit être fondée sur une connaissance préalable de l'objet de la recherche. En effet la description d'une image est capitale car elle constitue le transcodage des perceptions visuelles en un langage capable d'exprimer le contenu essentiel de celle-là ${ }^{3}$.

Toute personne ayant l'expérience de la miniature sait que l'image n'est intelligible que dans la mesure où l'on connaît le texte qu'elle illustre. L'image renvoie alors à un contenu verbal, qu'elle représente ou ne représente pas (ou très partiellement) suivant les cas. La question n'est donc pas de savoir si la connaissance du texte est nécessaire : elle l'est presque toujours. Une fois le problème de l'incompréhension de l'image surmonté, une autre difficulté survient : faut-il se contenter de décrire ce que l'on voit effectivement, au risque de ne pas saisir le sens de l'image ? Ou bien, comment éviter le danger inverse, qui consisterait à décrire le texte, plus que l'image elle-même ? La description d'une image, doit donc introduire :

a - la distinction et la désignation des différentes composantes d'une oeuvre illustrée (dans notre cas : légende, miniature, titre, texte)

b - la notion de rapports entre ces différentes composantes (par exemple la mise en page)

c - la description de ces composantes (en insistant surtout sur la description de la miniature)

Afin de mieux faire comprendre l'ambition et les limites de cette entreprise de description, je me référerai, au risque d'en simplifier excessivement la présentation, aux trois niveaux d'analyse définis par $\mathrm{E}$. Panofsky ${ }^{4}$ : la description pré-iconographique qui rend compte des motifs

(2) - Par là je veux tout simplement signaler que la description n'est qu'une étape de la recherche, sa fin étant l'analyse véritable de l'image.

(3) - À propos des différents langages utilisés actuellement pour la description des images et des problèmes liés à chacun (objectivité, monotonie...), voir les travaux de J. C. Gardin : "Methods for the Descriptive Analysis of Archeological Materials", American Antiquity, 32 (1967), pp. 13-30 ainsi que Archeological Constructs, an Aspect of Theoretical Archeology, Cambridge, 1979.

(4) - E. Panofski : Essais d'iconologie. Les thèmes humanistes dans l'art de la Renaissance, Paris, 1967. 
indépendamment de leur signification ; l'analyse iconographique qui reconnât le(s) thème(s) et, plus généralement, qui rend compte des significations conventionnelles dans un contexte donné ; et enfin l'interprétation iconologique qui dépasse l'identification des thèmes et interroge l'oeuvre comme symptôme, comme témoin des valeurs symboliques d'une civilisation.

À l'évidence, la description d'une image serait de faible profit si elle ne s'en tenait qu'au premier niveau. À l'inverse, on ne peut s'attendre à ce qu'elle s'engage dans une analyse iconologique. La description consiste donc en une saisie essentiellement iconographique de l'image. Plus précisément, on peut dire qu'elle se situe entre les deux premiers niveaux définis par E. Panofsky. En effet, la description d'une image ne peut pas se substituer à une véritable analyse qui nécessiterait une étude approfondie, d'une part de toutes les significations de l'image, et d'autre part, du contenu textuel.

\section{3 - LE “SYSTÈME D’ANALYSE DESCRIPTIVE”}

Comme il a été dit plus haut, le présent travail concerne l'élaboration d'un système d'analyse descriptive des images médiévales 5 .

(5) - Le présent système a été élaboré pour les besoins de mes recherches personnelles sur l'illustration de certains manuscrits grecs de contenu scientifique. Si je propose aujourd'hui ce système d'analyse c'est plus pour soulever des problèmes que pour proposer des solutions. En effet cette étude me parait plus utile pour servir d'exemple à d'autres démarches d'analyse beaucoup plus développées qu'en tant qu'outil de recherche qui pourra être utilisé dans l'état où il se trouve. Je dois également insister sur le fait que ce "système d'analyse descriptive» n'aurait pas vu le jour sans l'aide et l'expérience apportées par le «Groupe Image» de l'E.H.E.S.S. et plus spécialement par son directeur M. Jean-Claude Schmitt. Enfin j'aimerais citer certains travaux comme esquisse de bibliographie : J. Baschet : «Les vidéodisques des manuscrits de la Bibliothèque Vaticane et la réalisation d'une base de données iconographique», Arte medievale, 2ème série, 1 (1992), pp. 199-205. Th. ChatzidakisBacharas : «Projet d'un code pour l'analyse descriptive de l'iconographie byzantine» Dwdwvnh, 10 (1981), pp. 449-490 (cet article concerne un projet sur la peinture byzantine du Xle siècle qui, d'après mes connaissances, n'a jamais été réalisé). S. Dufrenne, C. Dumitrescu : «Autour du fichier descriptif et systématique des manuscrits byzantins décorés de la Bibliothèque nationale de Paris" Paleografia e codicologia greca, t. 1, pp. 321-323, Alessandria, 1991 (Actes du Ile colloque intern., Berlin-Wolfenbüttel, 17-21 oct. 1983). Fr. Garnier : Thésaurus iconographique. Système descriptif des représentations, Paris, 1984. M.R. Salomé : Code pour l'analyse des représentations figurées sur les vases grecs, Paris, 1980 et enfin Thesaurus des images médiévales pour la constitution de bases de données iconographiques, Paris, 1993. Ce dernier thesaurus a été publié par le "GROUPE IMAGE» de l'E.H.E.S.S. sous la direction de J. Baschet, J.-Cl. Bonne, A. Debert, M. Pastoureau et J.-Cl. Schmitt. Le livre de D. Muzerelle:Vocabulaire codicologique. Répertoire méthodologique des termes français relatifs aux manuscrits, Paris [Rubricae, 1], $1985 \mathrm{~m}$ 'a été également très utile. 
La description est divisée en quatre parties $(\mathrm{A}, \mathrm{B}, \mathrm{C} \text { et } \mathrm{D})^{6}$ dans un ordre hiérarchique, chacune se rapportant à un niveau plus fin de l'analyse descriptive des images.

La partie A comprend des informations générales concernant le manuscrit. Elle a été divisée en un certain nombre de rubriques qui sont les suivantes :

A.1 - Lieu : comporte le nom du lieu actuel de conservation du manuscrit : ville, nom de monastère, etc...

A. 2 - Bibliothèque : indique la bibliothèque où se trouve le manuscrit

A. 3 - Cote : comporte la cote actuelle du manuscrit et éventuellement le nom de la collection ou du fonds de la bibliothèque auquel appartient le manuscrit

A. 4 - Date : la date du manuscrit. Lorsque celle-ci n'est pas connue, elle sera indiquée en siècles. S'il y a des précisions à apporter, elles seront ajoutées après la date, entre parenthèses ;

A. 5 - Nombre de folios : le nombre de folios ${ }^{7}$ du manuscrit manuscrit

A. 6 - Format du manuscrit : les dimensions, en millimètres, du

La partie B comprend des informations générales concernant la décoration du folio et la miniature. Elle contient les rubriques suivantes :

B. $1-\mathrm{N}^{\circ}$ du folio : indique le folio où se trouve l'image à décrire

B. 2 - Emplacement : indique la position de la miniature par rapport au texte qu'elle illustre, par rapport à l'observateur et par rapport aux éventuelles autres images du même folio miniature

B. 3 - Format de la miniature : les dimensions, en millimètres, de la

B. 4 - Technique : on considère quatre catégories de techniques :

4. 1 - Dessin à la mine de plomb sur papier ( $Æ$ dessin)

4. 2 - Dessin à la mine de plomb sur parchemin (Æ dessin)

4. 3 - Peinture sur papier ( $Æ$ miniature)

(6) - Il faut souligner ici que j'ai utilisé le présent système descriptif dans sa version informatisée avec le logiciel de gestion de base de données Omnis 73 de Blyth Software Inc. Bien sûr d'autres logiciels (aussi bien dans le monde PC que dans le monde Macintosh) peuvent être utilisés; comme il s'agit d'un outil de travail personnel, donc utilisé pour traiter un nombre limité d'images, il a l'avantage par rapport aux autres systèmes descriptifs de ne nécessiter ni une programmation spécifique ni des péripheriques de stockage coûteux. Cependant, et pour des raisons évidentes j'ai été obligé de changer la présentation du système descriptif pour le rendre plus accessible.

(7) - Lorsque le chiffre donné est précédé du signe «pp.» cela signifie que le manuscrit n'est pas folioté mais paginé. 


\section{4 - Peinture sur parchemin ( $£$ miniature)}

B. 5 - Lettres capitulaires : indique l'éventuelle existence de lettres calligraphiques ou ornementales, se distinguant de l'écriture normalement utilisée pour le texte. Elles sont généralement employées pour inscrire les titres, les rubriques, les initiales, etc. Lors de la description il faut préciser leur nature. Dans le cas d'initiales d'un texte il faut préciser également si elles sont historiées ou ornées et, aussi, s'il s'agit des initiales des livres, des chapitres ou des paragraphes ${ }^{8}$

B. 6 - Bordure(s) : indique l'éventuelle existence de bordures au début d'un chapitre du texte

B. 7 - Encadrement : indique l'éventuelle présence de bandeaux décoratifs, de filets ou de bordures qui enserrent la miniature en limitant son champ

B. 8 - Fond : indique l'éventuelle existence d'un champ ou simplement d'un arrière-plan sur lequel se détachent les figures représentées ${ }^{9}$

B. 9 - Inscription : on transcrit et, si c'est nécessaire, on traduit la légende de l'image et le titre du chapitre illustré par celle-ci. Il faut également remarquer que, plusieurs fois, en absence de légende, le titre peut la remplacer.

La partie $\mathrm{C}$ contient des informations concernant l'ensemble de la miniature. Elle est divisée en quatre rubriques.

C. 1 - Thème : son but est de donner une compréhension immédiate de l'image sans entrer dans les détails. Autrement dit, il indique le sujet de la représentation en résumant les principaux aspects de celle-ci

C. 2 - Composant(s) : dans chaque image il y a un ou plusieurs composants explicitement reliés entre eux ;

C. 3. Composition : exprime la «syntaxe» (c'est-à-dire l'ordre de présentation) des principaux composants, ou éléments, de l'image ;

C. 4. Scène(s) : une image peut être composée d'une ou plusieurs scènes. Une scène est formée de plusieurs composants.

Une relation est explicite quand il y a :

\section{1. contact physique}

4. 2. interaction explicite

4. 3. interaction dans un sens plus large (rencontre, conversation, etc.) ou participation passive à l'action (spectateurs, témoins, etc.)

4. 4. participation à un même processus intransitif (marche, etc.).

(8) - Si l'initiale est dépourvue d'encadrement, de fond ainsi que de tout procédé décoratif quel qu'il soit, il s'agit d'une initiale nue.

(9) - Lors de la description si la miniature est peinte sans fond ni encadrement on peut utiliser l'expression "en champ libre". 
Nota bene : il est important de toujours indiquer le nombre de composants et le nombre de scènes (en précisant quelle est la scène principale et quelles sont les scènes secondaires).

La partie $\mathrm{D}^{10}$ comprend des informations concernant chaque composant de l'image. Elle est divisée en quatre grandes catégories :

- LES PERSONNAGES ET LEURS VÊTEMENTS (D. 1.)

- LES ANIMAUX ET LEUR HARNACHEMENT (D. 2.)

- LA VÉGÉtATION (D. 3.)

- LES AUTRES ÉLÉMENTS (D. 4.)

D. 1. 1. PERSONNAGES : leur description se fait en six étapes :

D. 1. 1. 1. Identité : les personnages sont généralement identifiés d'après une inscription ou selon des critères intrinsèques qui ne laissent aucun doute ;

D. 1. 1. 2. Posture : la position d'un personnage est déterminée par la façon dont son corps se tient dans l'espace. Les postures les plus fréquentes sont les suivantes:

1. à quatre pattes

2. agenouillé

3. assis

3. 1. sur le sol

3. 2. sur un animal

3. 3. sur un objet

4. couché

5. debout - immobile

debout - en marche

6. sur le dos

7. sur le ventre

8. tombé à terre

D. 1. 1. 3. Tête : pour la description de la tête nous indiquons la présence de la chevelure et de la barbe par les termes suivants :

1. calvitie partielle

2. calvitie totale

3. chevelure

(10) - Cette partie comporte différents termes (les descripteurs) propres à la description. Ces termes peuvent être modifiés ou augmentés selon les besoins du travail à effectuer. 
4. tête couverte (se rapporter à la rubrique «costume» : tête)

5. tonsure

6. barbe

7. imberbe

-La chevelure peut être :
a. courte
b. frisée
c. lisse
d. longue
e. mi-longue
f. ondulée

-La barbe peut être :
a. arrondie
b. bifide (en double pointe)
c. pointue

-La couleur ${ }^{11}$ de la chevelure et la couleur de la barbe sont indiquées par les termes suivants :
a. blanc
b. brun
c. châtain
d. noir
e. roux

-Angle de vue de la tête :

1. de dessus (vue supérieure)

\begin{abstract}
(11) - Il faut s'efforcer d'employer, pour la qualification des couleurs, des termes les plus simples possible afin que le lecteur puisse facilement identifier la couleur en question. En général la couleur d'un objet peut être décrite par trois caractéristiques : 1. la première est une caractéristique d'intensité et elle se traduit dans le langage naturel par les adjectifs «clair» ou «foncé». 2. la seconde caractéristique d'une couleur indique celle des couleurs pures dont la couleur considérée se rapproche le plus. Cette caractéristique se traduit par des adjectifs tels que : violet, bleu, vert, jaune, orange, rouge, pourpre (ou par une combinaison telle que : bleuvert, rouge-pourpre...). 3. la troisième caractéristique indique comment la couleur considérée se rapproche plus ou moins de la couleur pure correspondante. Dans le langage naturel, cette caractéristique se traduit par les adjectifs «pur»et «lavé de blanc».

Sur les couleurs au Moyen Age, voir les travaux de M. Pastoureau : Figures et couleurs. Étude sur la symbolique et la sensibilité médiévales, Paris, 1986 ainsi que Couleurs, images, symboles. Études d' histoire et d' anthropologie, Paris, 1989.
\end{abstract}


2. de dos

3. de face

4. de profil

5. de $3 / 4$

6. $3 / 4$ de dos

-On peut compléter les termes précédents par les termes suivants :
a. vers le bas (par rapport à l'observateur)
b. vers la droite
c. vers la gauche
d. vers le haut

D. 1. 1. 4. Corps : on indique l'axe du corps par rapport à la ligne horizontale (du cadre de l'illustration)
1. axe horizontal
2. axe oblique
3. axe vertical

-Angle de vue du corps :

1. de dessus (vue supérieure)

2. de dos

3. de face

4. de profil

5. de $3 / 4$

6. $3 / 4$ de dos

-On peut compléter les termes précédents par les termes suivants :

a. vers le bas (par rapport à l'observateur)

b. vers la droite

c. vers la gauche

d. vers le haut

D. 1. 1. 5. Bras : on indique les différents gestes en précisant chaque fois s'il s'agit d'un seul bras ( gauche ou droit) ou des deux.

D. 1. 1. 6. Jambes :

1. jambes droites

1. 1. jambes écartées

1. 2. jambes serrées

1. 3. l'une droite, l'autre en avant (ou en arrière)

2. jambes fléchies 
Nota Bene: les positions des bras et des jambes sont perçues généralement à travers les drapés des vêtements

D. 1. 2. COSTUME ${ }^{12}$ : les vêtements sont considérés en fonction de la partie du corps qu'ils recouvrent ; ainsi on distingue :

D. 1. 2. 1. Corps : pour la description des vêtements du corps on utilise les termes suivants :

1. bliaud

2. brogne

3. ceinture

4. gonelle

5. loros

6. pallium

7. tablion

8. trabéa

9. tunique

D. 1. 2. 2. Tête :

1. bonnet

2. chapel

3. collier

4. couronne

5. couvre-chef

D. 1. 2. 3. Jambes et pieds :

1. bamberges

2. bandes de lin

3. bottes brodées

4. bottes souples

5. chausses

6. nus

7. sandales

(12) - Par le terme "costume» j'entends l'ensemble de l'habillement porté par l'homme afin de couvrir et de parer son corps. Ce terme englobe donc, à part les vêtements, les chaussures, les couvre-chefs, les bijoux, les gants et les accessoires comme les ceintures, les bourses, les sacs, etc... 
D. 2. 1. ANIMAUX : leur description se fait en cinq étapes :

D. 2. 1. 1. Nature : on indique l'espèce à laquelle appartient l'animal, identifié généralement d'après une inscription ou selon des critères intrinsèques qui ne laissent aucun doute ;

D. 2. 1. 2. Posture ${ }^{13}$ : les termes exprimant les postures les plus fréquentes des animaux sont les suivants :

1. assis (sur les pattes arrière)

2. couché

2. 1. sur le côté

2. 2. sur les quatre pattes

3. debout - immobile

debout - en marche ${ }^{14}$

4. tombé par terre

D. 2. 1. 3. Tête

D. 2. 1. 4. Corps

pour la description de la tête et du corps nous indiquons l'angle de vue :

1. de dessus (vue supérieure)

2. de dos

3. de face

4. de profil

5. de $3 / 4$

6. $3 / 4$ de dos

-On peut compléter les termes précédents par les termes suivants :

a. vers le bas (par rapport à l'observateur)

b. vers la droite

c. vers la gauche

d. vers le haut

-la couleur du tégument ou du poil :
a. blanc
b. gris
c. marron
d. noir

(13) - Quand il s'agira de poisson on ne décrira pas sa posture.

(14) - Les termes utilisés pour décrire la posture d'un serpent seront les suivants : «allongé immobile» ou «allongé - mobile». 
e. roux

- On peut compléter les termes précédents par les termes suivants :

a. rayé

b. tacheté

-et l'axe de la tête et du corps par rapport à une ligne horizontale (du cadre de la miniature) :

1. axe horizontal

2. axe oblique

3. axe vertical

D. 2. 1. 5. Pattes ${ }^{15}$ :

1. pattes à segments articulés

2. pattes pliées

3. pattes tendues

3. 1. l'une droite, l'autre en avant (ou en arrière)

3. 2. pattes écartées

3. 3. pattes serrées

D. 2. 2. HARNACHEMENT : lorsqu'il y a des représentations d'animaux harnachés on décrit les harnais de diverses parties :

D. 2. 2. 1. Corps :

1. avaloire

2. barre de fesses

3 . collier

4. croupière

5. culeron

6. selle

7. sellette

D. 2. 2. 2. Tête :

1. anneau de filet

2. bride

3. mors

4. rênes

(15) - Il sera nécessaire de préciser s'il s'agit d'un quadrupède ou d'un bipède. En outre quand il s'agira d'un poisson on utilisera le terme "nageoires», et dans le cas d'un serpent cette rubrique ne fera pas partie de la description. 
D. 2. 2. 3. Pattes :

1. fer (à cheval, à boeuf, ...)

D. 3. VÉGÉTATION : la description se fait en sept étapes :

D. 3. 1. Nature : on indique l'espèce de la plante (ou de l'arbre) en question, identifiée généralement d'après une inscription ou selon des critères intrinsèques qui ne laissent aucun doute

D. 3. 2. Apparence : les termes les plus fréquents exprimant l'apparence des plantes (ou/et des arbres) sont les suivants :
1. couché
2. courbé
3. droit

-On peut compléter les termes précédents par les termes suivants :
a. épineux
b. feuillu
c. fleuri
d. fruitier
e. inerme
f. nu
g. ramifié

D. 3. 3. Feuilles ${ }^{16}$ : on indique la couleur, l'angle de vue et éventuellement on décrit la forme

D. 3. 4. Fleurs / D. 3. 5. Fruits : on indique la couleur pour chaque élément séparément

D. 3. 6. Tige $(s)^{17}$ : on indique la couleur et l'axe de la tige principale et des autres tiges ainsi que ceux des ramifications s'il y a lieu

D. 3. 7. Racines : dans le cas où les racines sont visibles on indique la couleur, l'angle de vue et éventuellement on décrit la forme.

D. 4. AUTRES ÉLÉMENTS : cette catégorie reste ouverte. En effet les composants décrits ici peuvent jouer un rôle primordial ou secondaire suivant les images considérées

(16) - Quand il s'agit d'un champignon on utilise le terme «chapeau».

(17) - Quand il s'agit d'un arbre on utilise les termes «tronc / branches» et quand il s'agit d'un champignon le terme "pied». 
D. 4. 1. Édifices: sous cette rubrique sont indiquées les représentations architecturales :

$$
\begin{aligned}
& \text { 1. église - extérieur } \\
& \text { église - intérieur }
\end{aligned}
$$

2. maison

3. palais

4. pavillon

5. tour

6. ville

D. 4. 2. Mobilier :

1. coussin

2. siège

2. 1. trône à dos

2. 2. trône simple

2. 3. tabouret

2. 4. autre type de siège

3. table

4. tapisserie

4. 1. lit

4. 2. matelas

D. 4. 3. Récipients :

1. boîte

2. corne à boire

3. cruche

4. sac

5. vase (pot)

D. 4. 4. Instruments :

1. instruments de guerre ou de chasse

1. 1. arc

1. 2. bouclier avec décor

1. 3. bouclier simple

1. 4. épée dégainée

1. 5. épée gainée

1.6. hache 
1. 7. lance

2. instruments de musique

2. 1. à cordes

2. 2. à percussion

2. 3. à vent

3. instruments médicaux

3. 1 . cautère

3. 2. couteau

3. 2. 1. bistouri

3. 3. pince

3. 4. pince tenaille

3. 5 . tarière

3. 5. 1. tarière avec arrêt

4. autres instruments

4. 1. bâton

4. 2. clés

D. 4. 5. Véhicules :

1. véhicule tiré par bateau

1. 1. bateau à voile

1. 2. bateau simple

2. véhicule tiré par un animal ${ }^{18}$

D. 4. 6. Nature : les éléments naturels sont décrits en trois étapes

1. Ciel :

1. 1. arc-en-ciel

1. 2. ciel uni (doré, bleu, autre couleur)

1. 3. en gloire (auréole)

1.4. étoiles

1. 5. lune

1. 6. rayons lumineux

1. 7. soleil

2. Terre :

2. 1. colline

(18) - On indique le nombre d'animaux, le genre de l'attelage (cheval, boeuf, etc.), la forme du chariot (caisse carrée, etc.) et le nombre de roues. 
2. 2. montagne

2. 3. pierre, caillou

2. 4. rocher

2. 5. sol plat

3. Eau :

3. 1. fleuve

3. 2. rivière

3. 3. source

D. 4. 7. Autres :

1. cavité artificielle

1. 1. puits

1. 2. tombeau

1. 2. 1. sarcophage

2. cavité naturelle

2. 1. alvéole

2. 2. grotte

3. colorants

3. 1. blanc de céruse

3. 2. khôl

3. 3. pourpre

4. liquide

4. 1. huile

4. 2. lait

4. 3. sang

4. 4. vin

5. substance minérale

5. 1. calcaire

5. 2. gypse

5. 3. litharge

5. 4. soufre.

En esquisse de conclusion j'aimerais remarquer qu'il est tout à fait possible que certaines rubriques ne paraissent pas nécessaires ou d'autres insuffisantes pour la description d'une image. En effet pour qu'il réponde aux différentes exigences et aux buts de la recherche effectuée dans le cadre d'un 
travail personnel, le présent système descriptif peut être modifié. Il est bien entendu que les différentes étapes de la description peuvent être interverties, de nouvelles rubriques peuvent être ajoutées, etc. Il faut souligner qu'il ne s'agit pas d'un instrument de travail prêt à être utilisé tel quel, mais, à l'opposé de la plupart des systèmes de description déjà publiés ${ }^{19}$, d'un système descriptif «ouvert».

(19) - Certains de ces systèmes ou projets de systèmes ont été brièvement décrits dans Le médiéviste et l'ordinateur, 26-27 (1993). 